The Journal of Neuroscience

https://jneurosci.msubmit.net

JN-RM-0083-21R2

Hand-selective visual regions represent how to grasp 3D tools: brain decoding during real actions

Stephanie Rossit, School of Psychology, University of East Anglia Ethan Knights, University of Cambridge

Courtney Mansfield, School of Psychology, University of East Anglia

Diana Tonin, School of Psychology, University of East Anglia Janak Saada, Department of Radiology, Norfolk and Norwich University Hospitals NHS Foundation Trust

Fraser Smith, University of East Anglia

Commercial Interest: 
A 3D-Printed Stimuli

Tool

Non-tool

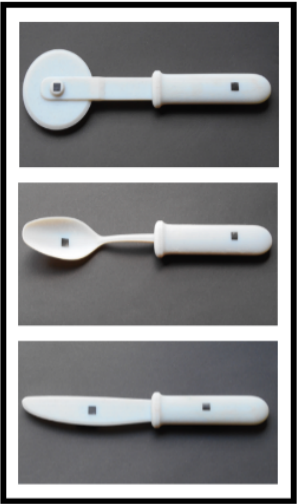

B

$\begin{array}{cccc}\text { Flex Illuminator } & \begin{array}{c}\text { Hand } \\ \text { Camera }\end{array} & \begin{array}{c}\text { Infrared } \\ \text { Source }\end{array} & \text { Fixation }\end{array}$

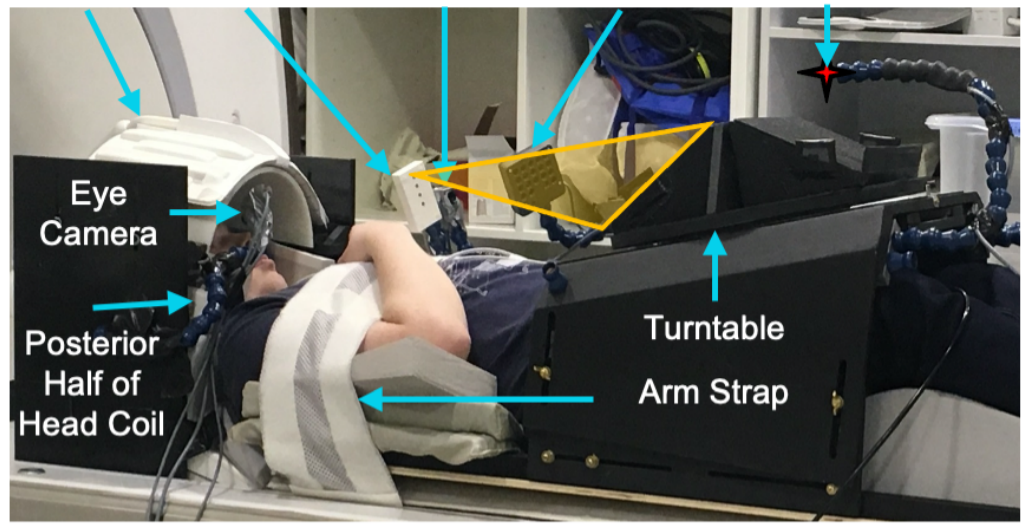

Real Action fMRI Setup

C

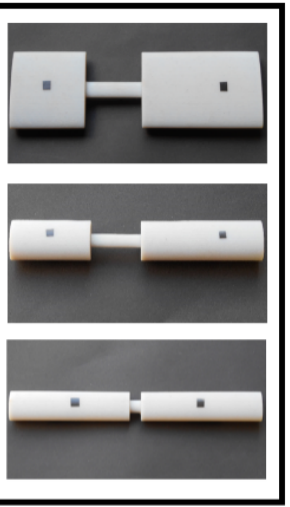

Real Action Experiment

(i))

"Left"

"Right"

.

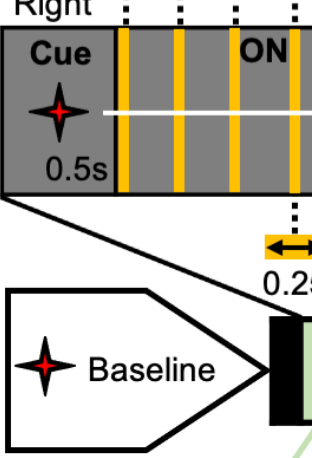

Right

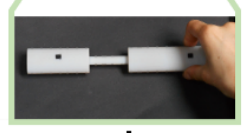

L vs.

Non-tool MVPA
Tool MVPA

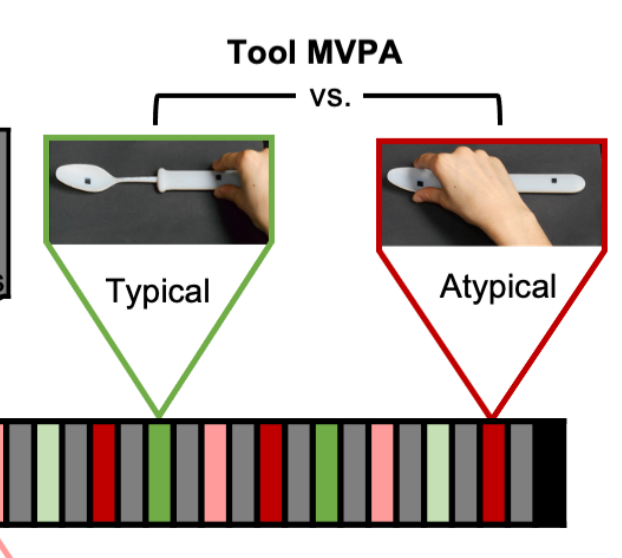

D

Visual Localizer Experiment

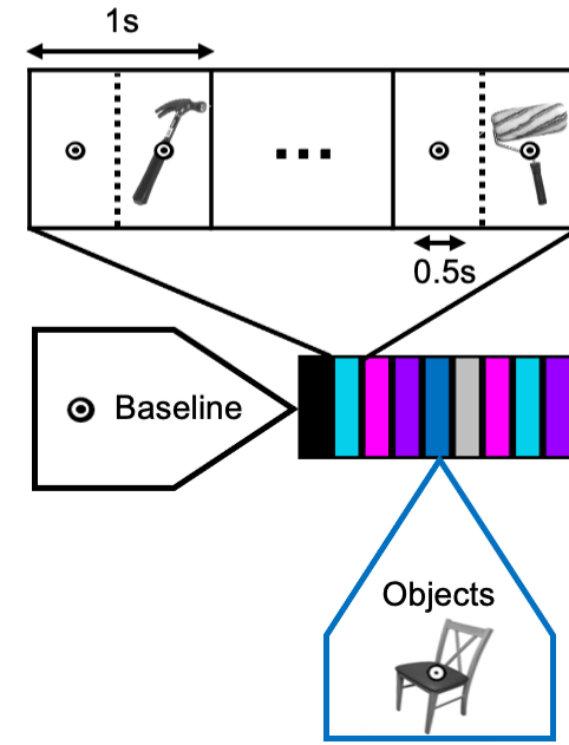

Left

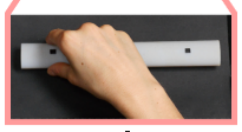

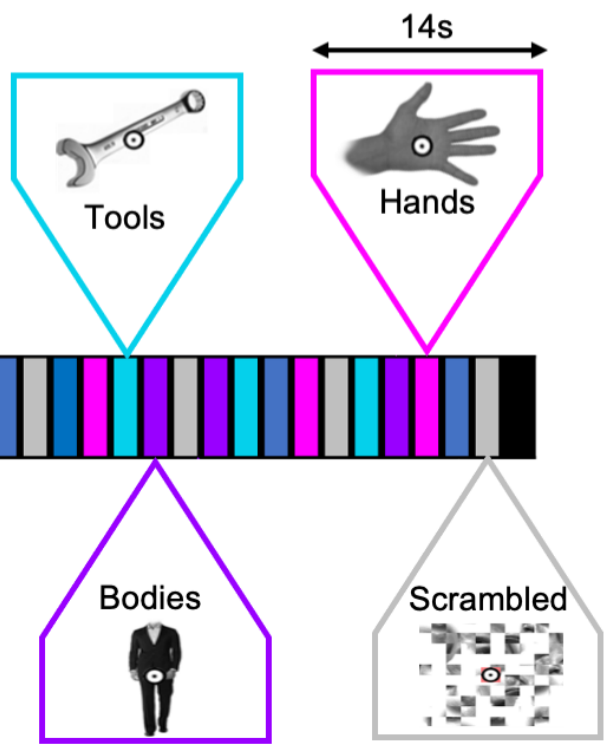

E Localizer ROIs

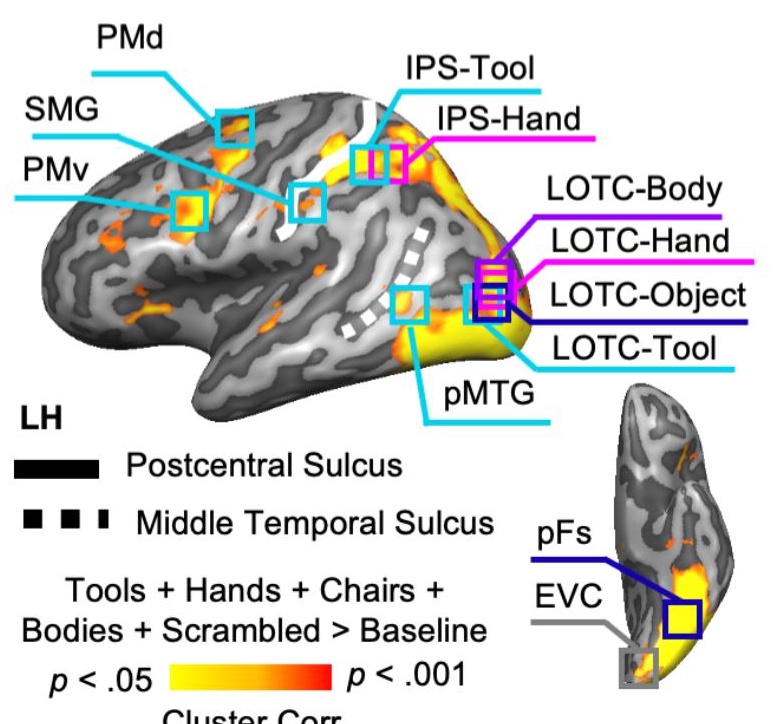




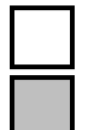

Tools: Typical vs. Atypical $\quad$ * $\mathrm{q}(\mathrm{FDR})<0.05$

Non-tools: Right vs. Left $\quad * \quad p<0.05$ (uncorrected)

... chance

Tools Hands Chairs Bodies Scrambled
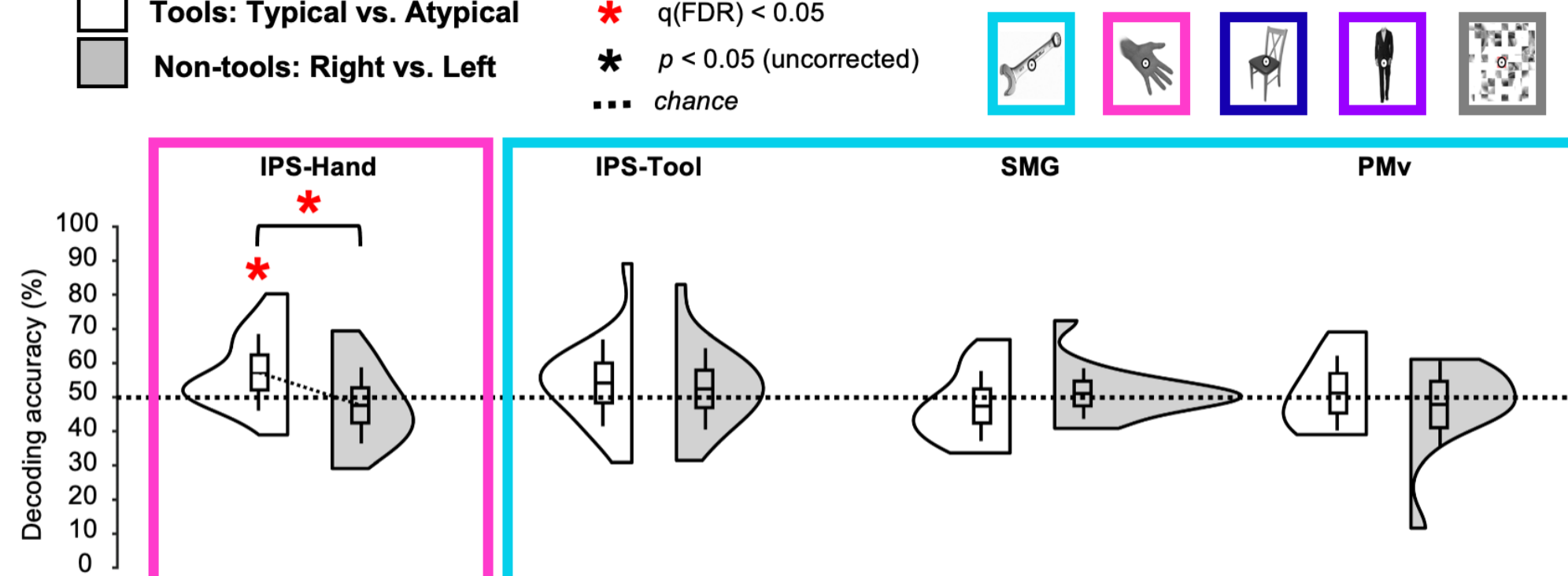

SMG

PMv
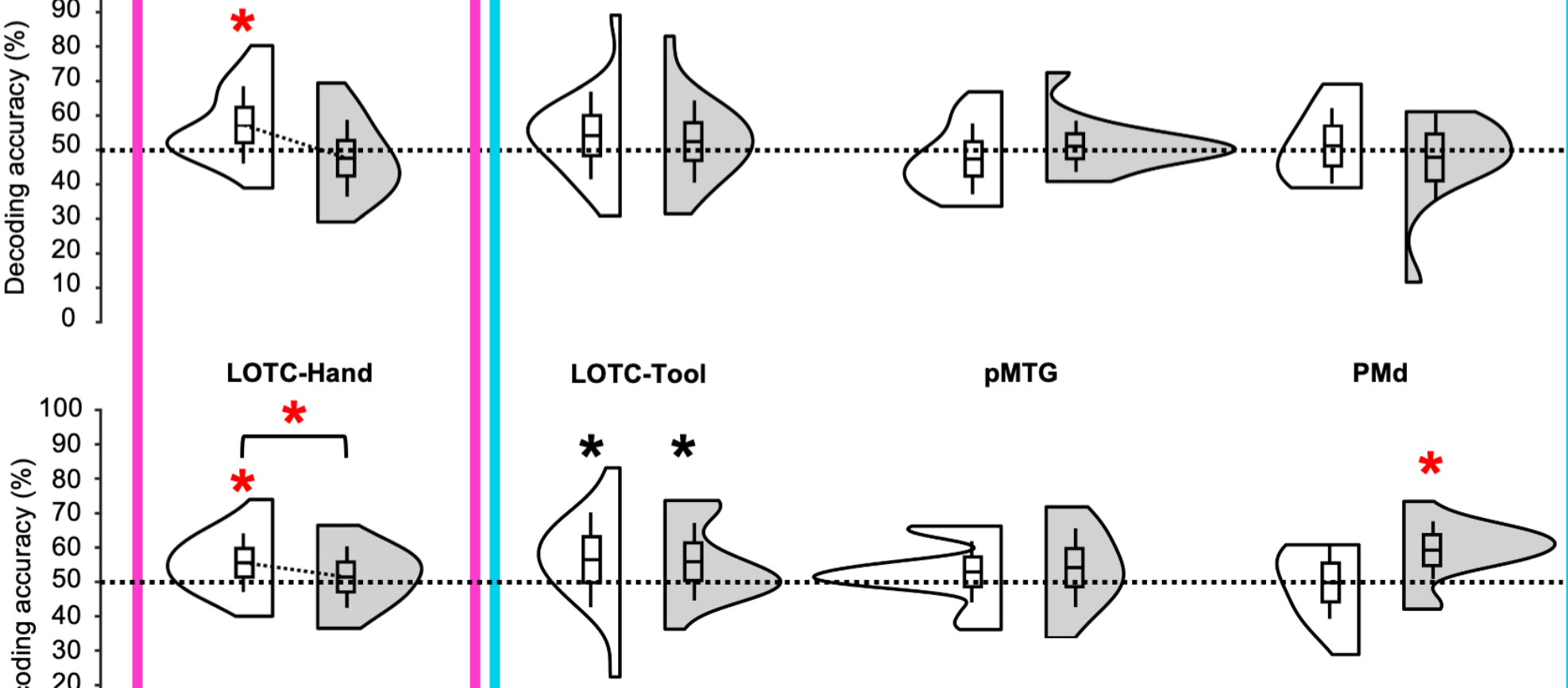

$\begin{array}{cc}8 & 20 \\ \square & 10 \\ 0 & 0\end{array}$
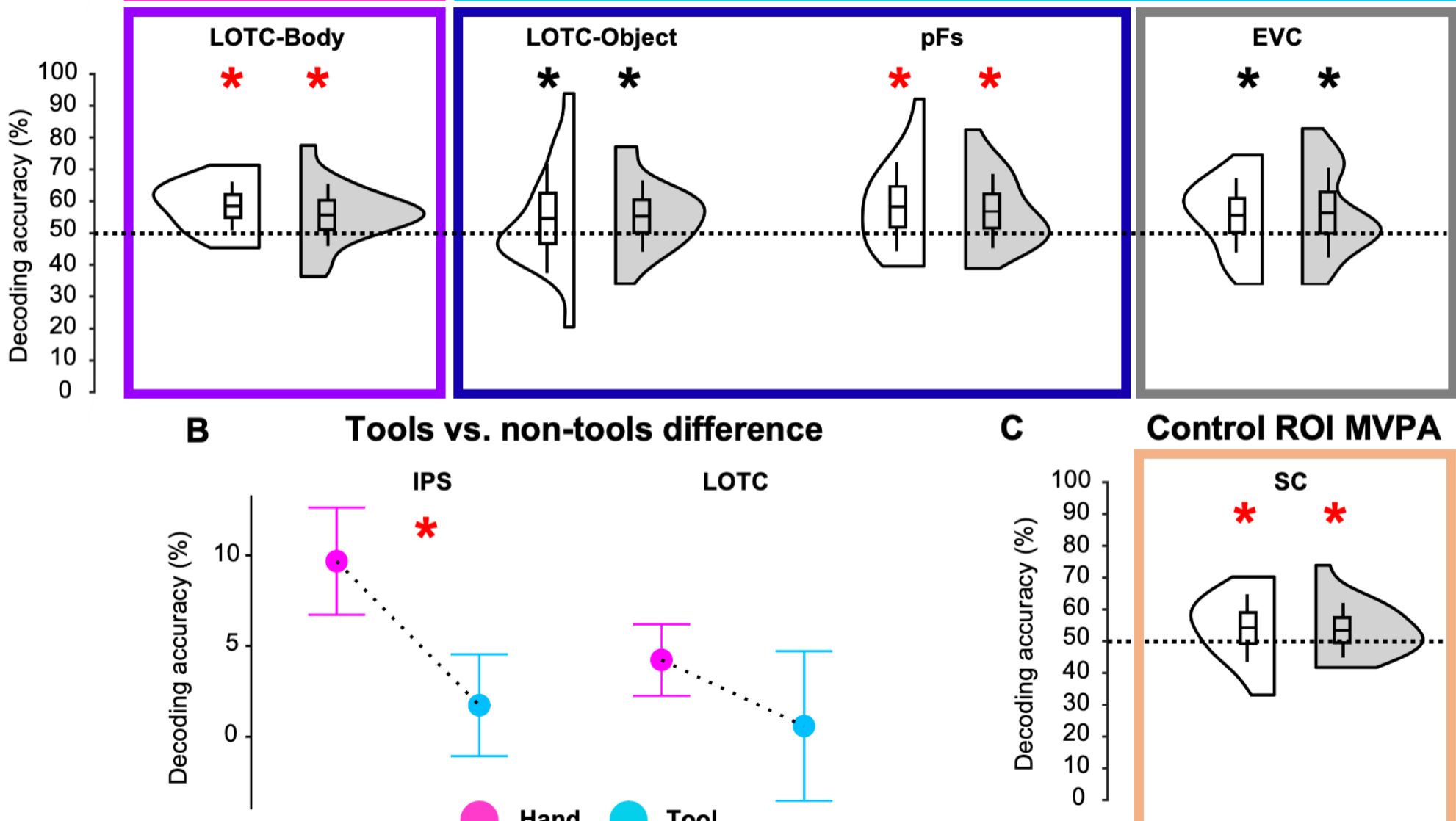

Control ROI MVPA 
Grip Size Control MVPA

A

Smaller
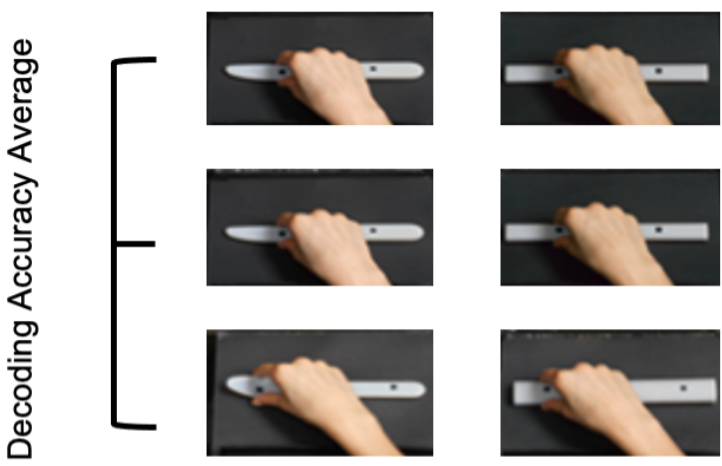

vs.

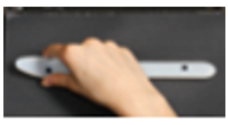

Larger

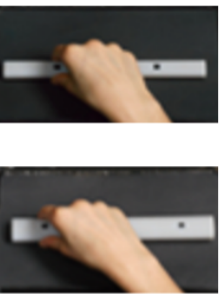

VS.
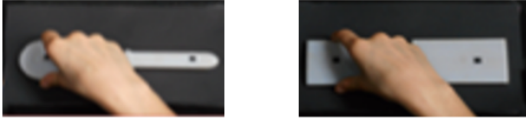

vs.
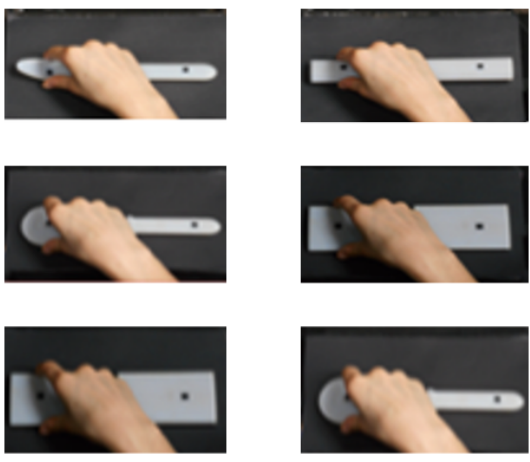

B

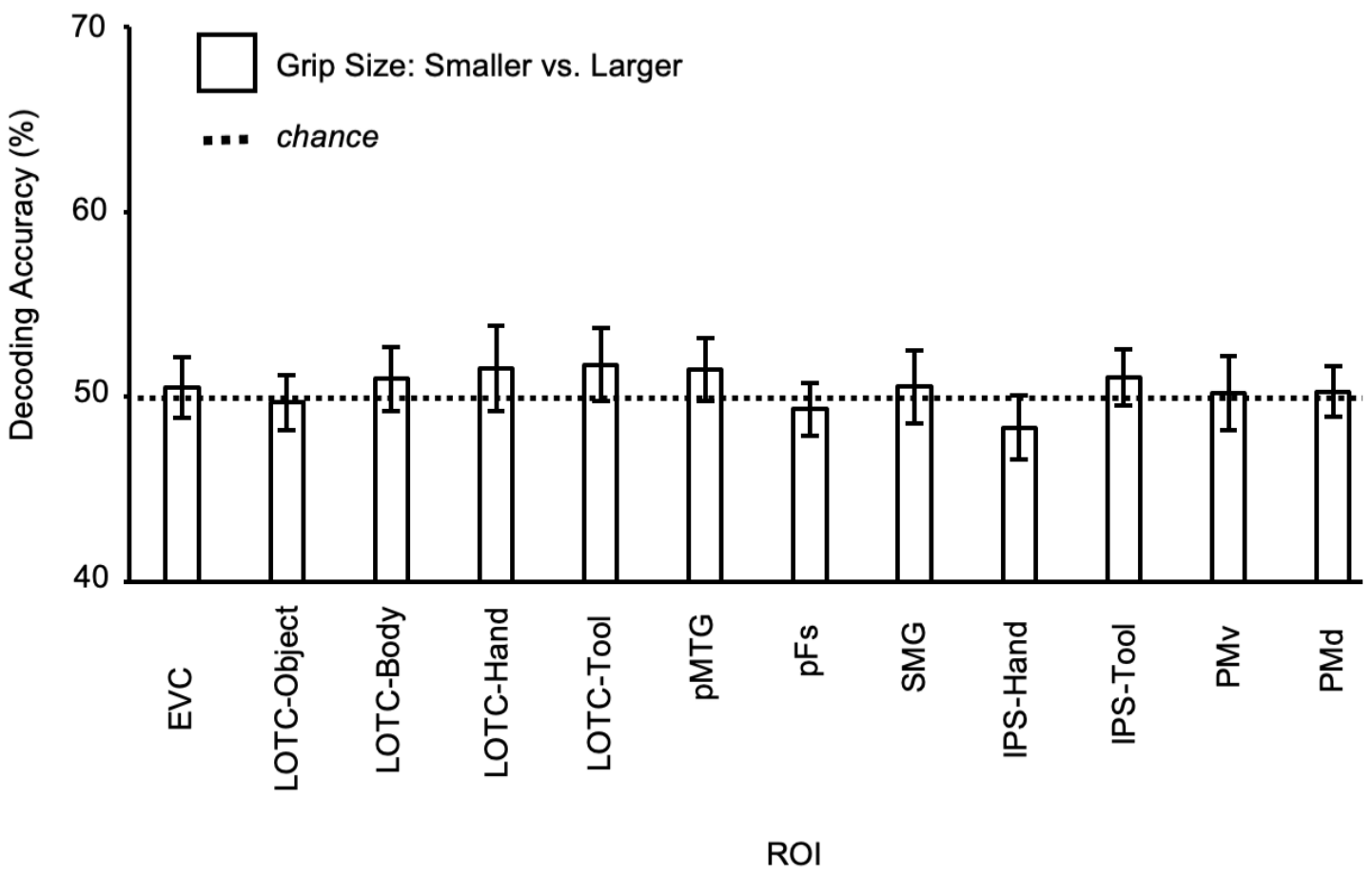


ROI Univariate Analysis

$\square$ Tool Typical $\square$ Non-tool Right
$\square$ Tool Atypical
Non-tool Left

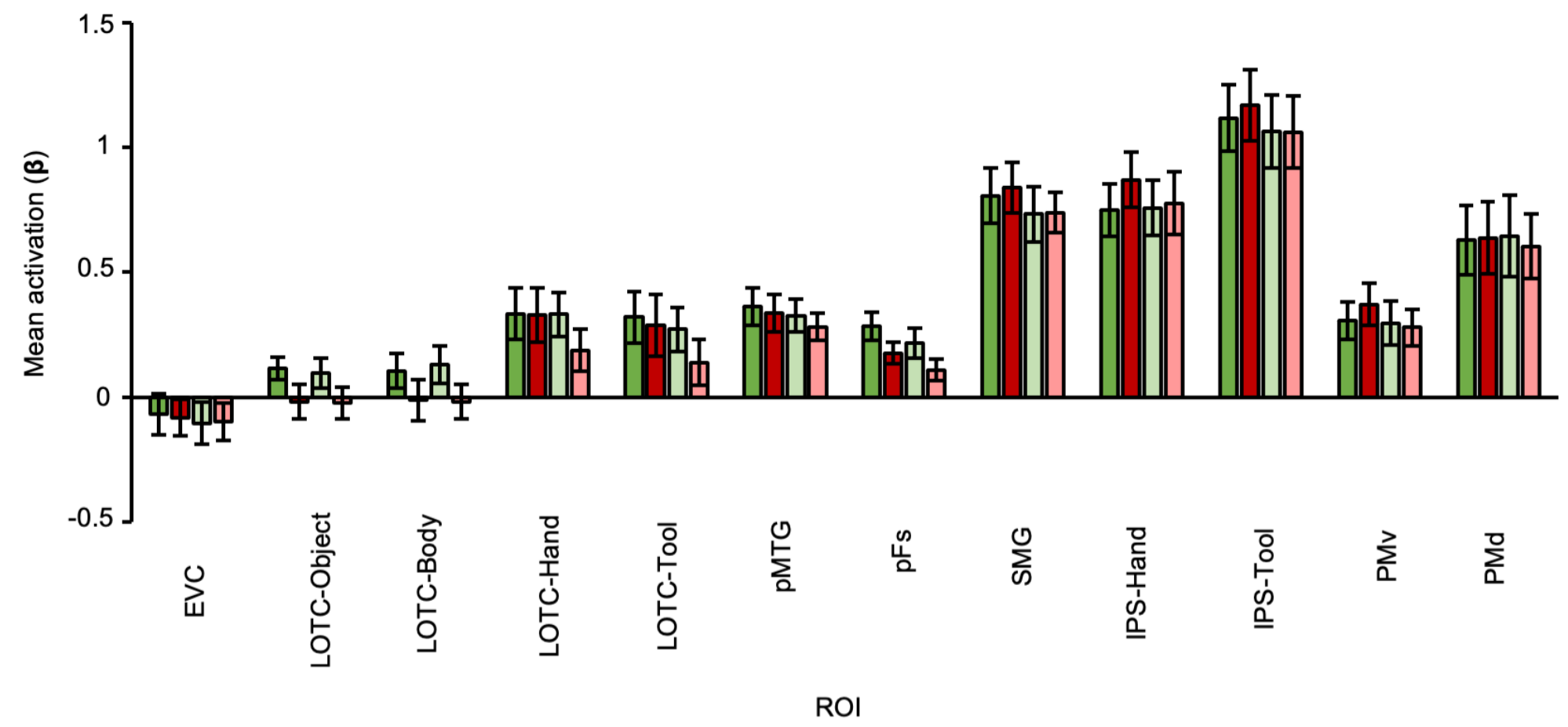


1 Title

2 Hand-selective visual regions represent how to grasp 3D tools: brain decoding during real

3 actions

4

5 Abbreviated title

6 Decoding tool grasping in hand-selective cortex

7

8 Authors \& Affiliations

9 Ethan Knights ${ }^{a}$, Courtney Mansfield ${ }^{b}$, Diana Tonin ${ }^{b}$, Janak Saada ${ }^{c}$, Fraser W. Smith ${ }^{b}, \&$

10 Stéphanie Rossit ${ }^{\mathrm{b}}$

$11{ }^{a}$ Medical Research Council Cognition \& Brain Sciences Unit, University of Cambridge,

12 Cambridge, CB2 7EF, UK

$13{ }^{\mathrm{b}}$ School of Psychology, University of East Anglia, Norwich, NR4 7TJ, UK

$14{ }^{\mathrm{C}}$ Department of Radiology, Norfolk and Norwich University Hospitals NHS Foundation Trust,

15 Norwich, NR4 7UY, UK

16

17 Corresponding author

18 Stephanie Rossit; s.rossit@uea.ac.uk

19

\section{Page/Word Counts}

21 Number of pages $=33$ pages

22 Figures $=4$

23 Tables $=1$

24 Number of words for Abstract $=250$ words

25 Number of words for Introduction= 643 words

26 Number of words for Discussion $=1500$ words

27

Conflict of interest statement

29 The authors declare no competing financial interests.

Acknowledgements

32 We thank Jenna Green, Richard Greenwood, Holly Weaver, Iwona Szymura and Emmeline

33 Mottram for support in data collection, Derek Quinlan for building the real action set-up, Stefania

34 Bracci for sharing the visual localizer stimuli and Annie Warman for comments on draft

35 manuscript. This work was funded by grant (184/14) from the BIAL Foundation awarded to S.

36 Rossit \& F.W. Smith. 


\section{Abstract}

38 Most neuroimaging experiments that investigate how tools and their actions are represented in

39 the brain use visual paradigms where tools or hands are displayed as 2D images and no real

40 movements are performed. These studies discovered selective visual responses in occipitotemporal and parietal cortices for viewing pictures of hands or tools, which are assumed to reflect action processing, but this has rarely been directly investigated. Here, we examined the responses of independently visually defined category-selective brain areas when participants grasped 3D tools ( $\mathrm{N}=20 ; 9$ females). Using real action $\mathrm{fMRI}$ and multi-voxel pattern analysis, we found that grasp typicality representations (i.e., whether a tool is grasped appropriately for use) were decodable from hand-selective areas in occipito-temporal and parietal cortices, but not from tool-, object-, or body-selective areas, even if partially overlapping. Importantly, these effects were exclusive for actions with tools, but not for biomechanically matched actions with control non-tools. In addition, grasp typicality decoding was significantly higher in hand than toolselective parietal regions. Notably, grasp typicality representations were automatically evoked even when there was no requirement for tool use and participants were naïve to object category (tool vs non-tools). Finding a specificity for typical tool grasping in hand-, rather than tool-, selective regions challenges the long-standing assumption that activation for viewing tool images reflects sensorimotor processing linked to tool manipulation. Instead, our results show that typicality representations for tool grasping are automatically evoked in visual regions specialised for representing the human hand, the brain's primary tool for interacting with the world.

\section{Significance Statement}

59 The unique ability of humans to manufacture and use tools is unsurpassed across the animal kingdom, with tool use considered a defining feature of our species. Most neuroscientific studies that investigate the brain mechanisms that support tool use, record brain activity while people simply view images of tools or hands and not when people perform actual hand movements with tools. Here we show that specific areas of the human visual system that preferentially process hands automatically encode how to appropriately grasp 3D tools, even when no actual tool use is required. These findings suggest that visual areas optimized for processing hands represent 
66 fundamental aspects of tool grasping in humans, such as which side they should be grasped for 67 correct manipulation. 
The emergence of handheld tools (e.g., a spoon) marks the beginning of a major discontinuity between humans and our closest primate relatives (Ambrose, 2001). Unlike other manipulable objects (e.g., books), tools are tightly associated with predictable motor routines (Johnson-Frey, 2004). A highly replicable functional imaging finding is that simply viewing tool pictures activates sensorimotor brain areas (Lewis, 2006), but what drives this functional selectivity? One popular idea is that this visually-evoked activation reflects the automatic extraction of information about the actions tools afford, like the hand movements required for their use (e.g., Martin et al., 1996; Fang \& He, 2005). Similarly, tool-selective visual responses in Supramarginal (SMG) or posterior Middle Temporal Gyri (pMTG) are often interpreted as indirect evidence that these regions are involved in real tool manipulation (e.g., Buxbaum et al., 2006; Bach et al., 2010). Nevertheless, we would never grasp a picture of a tool and, more importantly, finding spatially overlapping activation between two tasks does not directly imply that the same neural representations are being triggered (Dinstein et al., 2008; Martin, 2016). In fact, intraparietal activation for viewing tool pictures vs grasping shows poor correspondence (Valyear et al., 2007; Gallivan et al., 2013), questioning the long-standing assumption that visual tool-selectivity represents sensorimotor aspects of manipulation.

Curiously, the visual regions activated by viewing pictures of hands in the left Intraparietal Sulcus (IPS-Hand) and Lateral Occipital Temporal Cortex (LOTC-Hand) overlap with their respective tool-selective areas (IPS-Tool; LOTC-Tool; Bracci et al., 2012; 2013; 2016). Stimulus features often described to drive the organisation of category-selective areas, like form (Coggan et al., 2016), animacy (Konkle \& Caramazza, 2013) or manipulability (Mahon et al., 2007) poorly explain this shared topography because hands and tools differ on these dimensions. Instead, their overlap is suggested to result from a joint representation of high-level action information related to skilful object manipulation (Bracci et al., 2012; 2016; Striem-Amit et al., 2017), perhaps coding the function of hand configurations (Perini et al., 2014; Bracci et al., 2018). Arguably, the only way to directly test whether tool- or hand-selective visual areas carry information about tool actions is to examine their responses during real 3D tool manipulation. Yet, very few fMRI 
al., 2014; Styrkowiec et al., 2019). To date, only Gallivan et al. (2013) investigated real tool manipulation in visually defined tool-selective regions and showed that IPS-/LOTC-Tool are indeed sensitive to coarsely different biomechanical actions (reaching vs grasping) with a pair of tongs. However, it remains unknown whether hand-selective visual areas represent properties of real hand movements with 3D tools, like the way they are typically grasped for subsequent use. Here, an fMRI experiment involving real hand actions (Fig. 1) tested if visually defined hand- and tool-selective areas represented how to typically grasp 3D tools. Specifically, participants grasped 3D-printed tools in ways either consistent with their use (typical: by their handle) or not (atypical: by their functional-end; e.g., knife blade). As a control, non-tool bars (matched with the tools for elongation, width and depth; adapted from Brandi et al., 2014) were also grasped on their right or left sides to match as much as possible any biomechanical differences between typical and atypical actions. Multivoxel Pattern Analysis (MVPA) was used to assess whether different tool grasps (typical vs atypical) and non-tool grasps (right vs left), could be decoded from fMRI activity patterns within independent visually defined Regions of Interest (ROIs). Greater-than-chance decoding accuracy of typical vs atypical actions for tools,

112 but not control non-tools, was interpreted as evidence that an area contains high-level typicality 113 representations about how a tool should be grasped correctly for use (i.e., by its handle). This 114 pattern of findings was expected only for the tool- and hand-selective areas since these are 115 thought to support tool manipulation (e.g., Mahon \& Caramazza, 2009; Striem-Amit et al., 2017).

\section{$121 \quad$ Materials and Methods}

122 Participants. Twenty healthy participants (11 males) completed the real action fMRI experiment followed by a visual localizer experiment on a separate day. Data from one participant (male)

124 was excluded from statistical analysis due to excessive head movements during the real action experiment (i.e., translation and rotation exceeded $1.5 \mathrm{~mm}$ and $1.5^{\circ}$ rotation) leaving a total 
sample of 19 participants (mean age $=23$ years \pm 4.2 years; age range $=18-34$ ). All

127 participants had normal or corrected-to-normal vision, no history of neurological or psychiatric disorders, were right-handed (Oldfield, 1971) and gave written consent in line with procedures approved by the School of Psychology ethics committee at the University of East Anglia.

131 Real action 3D stimuli. Tool and non-tool object categories were designed (Autodesk Inc.) and

132 3D-printed (Objet30 Desktop) in VeroWhite material (Statasys): three common kitchen tools

133 (knife, spoon and pizzacutter) and three non-tool control bars (see Fig.1A). Objects were secured

134 to slots placed onto black pedestals used for stimulus presentation. Tools had identical handles

135 (length $\mathrm{x}$ width $\mathrm{x}$ depth dimensions of $11.6 \mathrm{~cm} \times 1.9 \mathrm{~cm} \times 1.1 \mathrm{~cm}$ ) with different functional-ends 136 attached $(\mathrm{knife}=10.1 \mathrm{~cm} \times 1.9 \mathrm{~cm} \times 0.2 \mathrm{~cm} ;$ spoon $=10.1 \mathrm{~cm} \times 4.1 \mathrm{~cm} \times 0.7 \mathrm{~cm} ;$ pizzacutter $=$ $137 \quad 10.1 \mathrm{~cm} \times 7.5 \mathrm{~cm} \times 0.2 \mathrm{~cm}$ ). To avoid motor or visual confounds, tools and non-tool pairs were 138 carefully matched in terms of visual properties and kinematic requirements as much as possible.

139 Specifically, non-tools were comprised of three cylindrical shapes (adapted from Brandi et al., 140 2014) with handle, neck and functional-end dimensions matched to each tool they controlled for, 141 ensuring that grip size was matched between tool and non-tool pairs. In addition, all objects had 142 small black stickers placed at pre-specified locations to indicate grasp points, ensuring that grasp position/reach-distance were identical between tool and non-tool pairs regardless of side to be grasped. To avoid familiarity confounds between tools and non-tool control stimuli we chose to use bars instead of scrambled tools and thus, our control non-tools were familiar, but had no specific associated function. Furthermore, each tool and non-tool pair were carefully matched for elongation so that any differences between conditions could not be explained by low-level shape preferences (e.g., Sakuraba et al., 2012; Brandi et al., 2014).

150 Real action setup and apparatus. Participants were scanned in complete darkness using a 151 head-tilted configuration that allowed direct viewing of the workspace and 3D stimuli without the use of mirrors (Fig. 1B) by tilting the head coil $\sim 20^{\circ}$ and padding the underside of each

153 participants heads with foam cushions (NoMoCo Pillow, La Jolla, CA, USA). Objects were placed 154 by an experimenter on a turntable above the participant's pelvis and were only visible when 
155 illuminated (e.g., Fernández-Espejo et al., 2015; Fig. 1B). All stimuli were mounted such that they

156 were aligned with participants' midlines, never changed position while visible and were tilted

157 away from the horizontal at an angle $\left(\sim 15^{\circ}\right)$ to maximize visibility and grasp comfort. For stimulus

158 presentation, the workspace and object were illuminated from the front using a bright white Light

159 Emitting Diode (LED) attached to a flexible plastic stalk (Loc-line, Lockwood Products; Fig. 1B).

160 To control for eye movements, participants were instructed to fixate a small red LED positioned

161 above and behind objects such that they appeared in the lower visual field (Rossit et al., 2013).

162 Throughout the experiment, participants' right eye and arm movements were monitored online

163 and recorded using two MR-compatible infrared-sensitive cameras (MRC Systems $\mathrm{GmbH}$ ) to

164 verify that participants performed the correct grasping movement (hand camera positioned over

165 the left shoulder; Fig. 1B) and maintained fixation (eye camera beside the right eye; Fig. 1B).

166 The likelihood of motion artefacts related to grasping was reduced by restraining the upper-right

167 arm and providing support with additional cushions so that movements were performed by flexion

168 around the elbow only (Culham, 2006). Auditory instructions were delivered to the participants

169 through earphones (Sensimetrics MRI-Compatible Insert Earphones Model S14, USA). At the

170 beginning of the real action session, participant setup involved adjusting the exact position of: 1)

171 stimuli and the hand to ensure reachability (average grasping distance between the "home"

172 position and object $=43 \mathrm{~cm}$ ), 2) the illuminator to equally light all objects, 3 ) the fixation LED to

173 meet the natural line of gaze (average distance from fixation to bridge nose $=91 \mathrm{~cm}$; visual angle

$174=\sim 20^{\circ}$ ) and 4) the infrared-sensitive eye and hand cameras to monitor eye and hand movement

175 errors. The experiment was controlled by a Matlab script (The MathWorks, USA R2010a) using

176 the Psychophysics Toolbox (Brainard, 1997).

178 Real action experimental paradigm. We used a powerful block-design fMRI paradigm, that

179 maximised the contrast-to-noise ratio to generate a reliable estimate of the average response

180 pattern (Mur et al., 2009) and improved detection of blood oxygenation level-dependent (BOLD)

181 signal changes without significant interference from artefacts during overt movement (Birn et al.,

182 2004). A block began with an auditory instruction ('Left' or 'Right'; 0.5s) specifying which side of

183 the upcoming object to grasp (Fig. 1C). During the ON-block (10s), the object was briefly 
184 illuminated for 0.25 s five consecutive times (within $2 \mathrm{~s}$ intervals) cueing the participant to grasp

185 with a right-handed precision grip (i.e., index finger and thumb) along the vertical axis. Between 186 actions, participants returned their hand to a "home" position with their right hand closed in a fist 187 on their chest (see Fig. 1B). This brief object flashing presentation cycle during ON-blocks has 188 been shown to maximise the signal-to-noise ratio in previous perceptual decoding experiments 189 (Kay et al., 2008; Smith \& Muckli, 2010) and eliminates the sensory confound from viewing hand 190 movements (Rossit et al., 2013; Monaco et al., 2015). An OFF-block (10s) followed the 191 stimulation block where the workspace remained dark and the experimenter placed the next stimulus. A single fMRI run included 16 blocks involving the four grasping conditions (i.e., typical

193 tool, atypical tool, right non-tool and left non-tool) each with three repetitions (one per exemplar; 194 every object was presented twice and grasped on each side once). An additional tool (whisk) and a non-tool object were presented on the remaining four blocks per run, but not analysed as they

196 were not matched in dimensions due to a technical problem (the original control non-tool for the 197 whisk was too large to allow rotation of the turntable within the scanner bore). On average participants completed six runs (minimum five, maximum seven) for a total of 18 repetitions per

199 grasping condition. Block orders were pseudorandomised such that conditions were never 200 repeated (two-back) and were preceded an equal number of times by other conditions. Each functional scan lasted 356s, inclusive of start / end baseline fixation periods (14s). Each experimental session lasted $\sim 2.25$ hours (including setup, task practice and anatomical scan).

203 Prior to the $\mathrm{FMRI}$ experiment, participants were familiarised with the setup and practiced the grasping tasks in a separate lab session (30 minutes) outside of the scanner. The hand and eye movement videos were monitored online and offline to identify error trials. Two runs (of two separate participants) from the entire dataset were excluded from further analysis. In one of these blocks the participant failed to follow the grasping task instructions correctly (i.e.,

208 performing alternated left and right grasps) and for the remaining block another participant did 209 not maintain fixation (i.e., made downward saccades toward objects). In the remaining runs that 210 were analysed, participants made performance errors in $<1 \%$ of experimental trials. The types of 211 errors included: not reaching (3 trials, 2 participants), reaching in the wrong direction ( 1 trial, 1 212 participant) and downward eye saccades (5 trials, 3 participants). A one-way repeated measures 
213 ANOVA with 12 levels (i.e., the six exemplars across both left vs right grasping conditions)

214 showed that the percentage of errors were equally distributed amongst trial types regardless of whether the percentage of hand and eye errors were combined or treated separately (all p's >

$2160.28)$

217 Crucially, since the tools' handles were always oriented rightward, the right and left tool

218 trials involved grasping tools either by their handle (typical) or functional-end (atypical),

219 respectively. On the other hand, grasping non-tools did not involve a typical manipulation but

220 only differed in grasp direction with right vs left grasps (Fig. 1C). We chose to present rightward

221 oriented tool handles only, rather than alternate object orientation randomly between trials, to

222 reduce total trial numbers (scanning times was already quite extensive with set-up) and due to

223 technical limitations (i.e., the turntable's rotation direction was fixed and it was difficult for the

224 experimenter to manipulate tool orientation in the dark). Nevertheless, by comparing the

225 decoding accuracies for each region between tool and non-tool grasps (which were matched for

226 biomechanics) we ruled out the possibility that our typically manipulation simply reflected grasp

227 direction. Specifically, we took the conservative approach that for an area to be sensitive to tool

228 grasping typicality, it should not only show greater-than-chance decoding for typical vs atypical

229 actions with tools (i.e., typicality), but also that the typicality decoding accuracy should be

230 significantly greater than accuracy for biomechanically matched actions with our control non-tools

231 (i.e. right vs left actions with non-tools).

233 Visual Localizer. On a separate day from the real action experiment, participants completed a

234 Bodies, Chairs, Tools and Hands (BOTH) visual localizer (adapted from Bracci et al., 2012; 2013;

235 2016) using a standard coil configuration (see MRI acquisition for details). Two sets of exemplar

236 images were selected from previous stimuli databases (Bracci et al., 2012; 2013; 2016) that were

237 chosen to match, as much as possible, the characteristics within the tool (i.e., identity \&

238 orientation), body (i.e., gender, body position \& amount of skin shown), hand (i.e., position \&

239 orientation) and chair (i.e., materials, type \& style) categories. Using a mirror attached to the

240 head coil, participants viewed separate blocks (14s) of 14 different grayscale 2D pictures from a

241 given category ( $400 \times 400$ pixels; $0.5 \mathrm{~s})$. Blank intervals separated individual stimuli $(0.5 \mathrm{~s})$ and 
scrambled image blocks separated cycles of the four randomised category blocks (Fig. 1D).

243 Throughout, participants fixated a superimposed bullseye on the centre of each image and, to encourage attention, performed a one-back repetition detection task where they made a righthanded button press whenever 2 successive photographs were identical. The 2D images stimuli were presented with an LCD projector (SilentVision SV-6011 LCD, Avotech Inc.). A single fMRI run included 24 category blocks (6 reps per condition) with blank fixation baseline periods (14s) at the beginning and the end of the experiment. Each localizer scan lasted $448 \mathrm{~s}$ and, on average, participants completed 4 runs (minimum 3, maximum 4) for a total of 24 reps per condition. The entire localizer session lasted $\sim 50$ minutes after including the time taken to acquire a high-resolution anatomical scan and setup participants.

MRI Acquisition. The BOLD fMRI measurements were acquired using a 3T wide bore GE-750 Discovery MR scanner at the Norfolk \& Norwich University Hospital (Norwich, UK). To achieve a good signal to noise ratio during the real action fMRI experiment, the posterior half of a 21 channel receive-only coil was tilted and a 16-channel receive-only flex coil was suspended over the anterior-superior part of the skull (see Fig. 1B). A T2*-weighted single-shot gradient EchoPlaner Imaging (EPI) sequence was used throughout the real action experiment to acquire 178 functional MRI volumes (Time to Repetition $(T R)=2000 \mathrm{~ms}$; Voxel Resolution $(\mathrm{VR})=3.3 \times 3.3 \mathrm{x}$ $3.3 \mathrm{~mm}$; Time to Echo $(\mathrm{TE})=30 \mathrm{~ms}$; Flip Angle $(\mathrm{FA})=78^{\circ}$; Field of View $(\mathrm{FOV})=211 \times 211 \mathrm{~mm}$; Matrix Size $(M S)=64 \times 64$ ) that comprised 35 oblique slices (no gap) acquired at $30^{\circ}$ with respect to $\mathrm{AC}-\mathrm{PC}$, to provide near whole brain coverage. A T1-weighted anatomical image with 196 slices was acquired at the beginning of the session using BRAVO sequences (TR $=2000 \mathrm{~ms}$; $\mathrm{TE}=30 \mathrm{~ms} ; \mathrm{FOV}=230 \mathrm{~mm} \times 230 \mathrm{~mm} \times 230 \mathrm{~mm} ; \mathrm{FA}=9^{\circ} ; \mathrm{MS}=256 \times 256 ;$ Voxel size $=0.9 \times 0.9$ $\times 0.9 \mathrm{~mm})$.

For visual localizer sessions, a full 21-channel head coil was used to obtain 224 functional MRI volumes (Time to Repetition $(T R)=2000 m s$; Voxel Resolution $(V R)=3.3 \times 3.3 \times$ 3.3mm; Time to Echo $(\mathrm{TE})=30 \mathrm{~ms}$; Flip Angle $(\mathrm{FA})=78^{\circ}$; Field of View $(\mathrm{FOV})=211 \times 211 \mathrm{~mm}$; Matrix Size $(M S)=64 \times 64)$. A high resolution T1-weighted anatomical image with 196 slices was acquired before the localizer runs $(\mathrm{TR}=2000 \mathrm{~ms} ; \mathrm{TE}=30 \mathrm{~ms} ; \mathrm{FOV}=230 \mathrm{~mm} \times 230 \mathrm{~mm} \times$ 
$271230 \mathrm{~mm} ; \mathrm{FA}=9^{\circ} ; \mathrm{MS}=256 \times 256 ;$ Voxel size $\left.=0.9 \times 0.9 \times 0.9 \mathrm{~mm}\right)$. Localizer datasets for two

272 participants were retrieved from another study from our group (Rossit et al., 2018) where the 273 identical paradigm was performed when acquiring volumes using a Siemens whole-body 3T

274 MAGNETOM Prisma fit scanner with a 64-channel head coil and integrated parallel imaging 275 techniques at the Scannexus imaging centre (Maastricht, The Netherlands) and comparable 276 acquisition parameters (Functional scans: $\mathrm{TR}=2000 \mathrm{~ms} ; \mathrm{TE}=30 \mathrm{~ms} ; \mathrm{FA}=77^{\circ} ; \mathrm{FOV}=216 \mathrm{~mm}$;

$277 \mathrm{MS}=72 \times 72 ;$ Anatomical scan: 1 1-weighted anatomical image: $\mathrm{TR}=2250 \mathrm{~ms} ; \mathrm{TE}=2.21 \mathrm{~ms} ; \mathrm{FA}$ $\left.278=9^{\circ} ; \mathrm{FOV}=256 \mathrm{~mm} ; \mathrm{MS}=256 \times 256\right)$.

279

280 Data Preprocessing. Preprocessing and ROI definitions were performed using BrainVoyager 281 QX (version 2.8.2) (Brain Innovation, Maastricht, The Netherlands). BrainVoyager's 3D motion correction (sinc interpolation) aligned each functional volume within a run to the functional volume acquired closest in time to the anatomical scan (e.g., Rossit et al., 2013). Slice scan time correction (ascending and interleaved) and high-pass temporal filtering (2 cycles/run) was also performed. Functional data were superimposed on to the anatomical brain images acquired during the localizer paradigm that were previously aligned to the plane of the anterior-posterior commissure and transformed into standard stereotaxic space (Talairach, \& Tournoux, 1988). Excessive motion was screened by examining the time-course movies and motion plots created with the motion-correction algorithms for each run. No spatial smoothing was applied.

291 (i.e., Bodies, Objects, Tools, Hands and Scrambled) in a single-subject general linear model

292 (GLM). Predictors were created from boxcar functions that were convolved with a standard $2 y$ 293 model of the hemodynamic response function (Boynton et al., 1996) and aligned to the onset of 294 the stimulus with durations matching block length. The baseline epochs were excluded from the 295 model, and therefore, all regression coefficients were defined relative to this baseline activity.

296 This process was repeated for the real action experiment, using 16 separate predictors for each

297 block of stimulation independently per run (12 exemplars - knife typical, knife atypical, spoon 298 typical etc. plus 4 foil trials) and 6 motion regressors (confound predictors). These estimates 299 (beta weights) from the real action experiment were used as the input to the pattern classifier. 
300 Visual Localizer Regions of interest (ROIs). Twelve visual ROls were defined at the individual

301 participant level from the independent BOTH localizer data by drawing a cube (15 voxels $\left.{ }^{3}\right)$

302 around the peak of activity from previously reported volumetric contrasts (see list below; Fig. 1E;

303 Table 1) set at a threshold of $p<.005$ (Gallivan et al., 2013) or, if no activity was identified, of $p<$

304.01 (Bracci et al., 2016). In cases where no activity was observed, the ROI was omitted for that

305 participant (see Table 1). Given the predominantly left lateralised nature of tool-processing

306 (Lewis, 2006), all individual participant ROls were defined in the left hemisphere (Bracci et al.,

307 2012; 2013; 2016; Peelen et al., 2013). Six tool-selective ROls commonly described in left

308 frontoparietal and occipitotemporal cortices were identified by contrasting activation for tool

309 pictures vs other object pictures (IPS-Tool; SMG; dorsal and ventral Premotor Cortex (PMd;

310 PMv), LOTC-Tool; pMTG; Martin et al., 1996; Grafton et al., 1997). Moreover, two hand-selective

311 ROIs were identified in LOTC (LOTC-Hand) and IPS (IPS-Hand) by contrasting activation for

312 hand pictures vs pictures of other body parts (Bracci et al., 2012; 2016; 2018; Peelen et al.,

313 2013; Palser \& Cavina-Pratesi, 2018). Additionally, we defined a body-selective (LOTC-Body;

314 Bodies > Chairs; Bracci \& de Beeck, 2016), two object-selective ROls (LOTC-Object; posterior

315 Fusiform, pFs; Chairs > Scrambled; Bracci \& de Beeck, 2016; Hutchison et al., 2014) and an

316 Early Visual Cortex ROI (EVC; All Categories > Baseline; Bracci \& de Beeck, 2016). The ROI

317 locations were verified by a senior author (S.R.) with respect to the following anatomical

318 guidelines and contrasts:

319 - Lateral Occipitotemporal Cortex-Object selective (LOTC-Object) - (Chairs > Scrambled)

320 (Hutchison et al., 2014; Bracci \& de Beeck, 2016) - defined by selecting the peak of

321 activation near the Lateral Occipital Sulcus (LOS; Hutchison et al., 2014; Bracci \& de

$322 \quad$ Beeck, 2016; Malach et al., 1995; Grill-Spector et al., 1999; 2001).

323 - Lateral Occipitotemporal Cortex-Body selective (LOTC-Body) - (Bodies > Chairs) (Bracci

324 \& de Beeck, 2016) - defined by selecting the peak of activation near the LOS and inferior

325 to the left Extrastriate Body Area (EBA; Valyear \& Culham, 2010) which was identified by

326 the contrast ((Bodies + Hands) > Chairs) (adapted from Bracci, et al., 2010; ((Whole

327 Bodies + Body Parts $)>($ Hands + Chairs $)))$. EBA was not included in the analysis. 
- Lateral Occipitotemporal Cortex-Hand selective (LOTC-Hand) - ((Hands > Chairs) AND (Hands > Bodies)) (Bracci \& de Beeck, 2016) - defined by selecting the peak of activation near the LOS. These were often anterior to LOTC-Body (Bracci et al., 2010; 2016).

- Lateral Occipitotemporal Cortex-Tool selective (LOTC-Tool) - (Tools > Chairs) (Bracci, et al., 2012; Hutchison et al., 2014) - defined by selecting the peak of activation near the LOS. These often closely overlapped LOTC-Hand (Bracci, et al., 2012).

- Posterior Middle Temporal Gyrus (pMTG) - (Tools > Chairs) (Hutchison, et al., 2014; Valyear \& Culham, 2010) - defined by selecting the peak of activation on the PMTG, more lateral, ventral and anterior to EBA (Hutchison et al., 2014). We selected the peak anterior to the Anterior Occipital Sulcus (AOS), as the MTG is in the temporal lobe and the AOS separates the temporal from the occipital (Damasio, 1995).

- Posterior Fusiform Sulcus (pFs) - (Chairs > Scrambled) (Hutchison, et al., 2014) - defined by selecting the peak of activation in the posterior aspect of the fusiform gyrus, extending into the occipitotemporal sulcus (Hutchison, et al., 2014).

- Intraparietal Sulcus-Hand selective (IPS-Hand) - (Hands > Chairs) (Bracci, et al. 2016; Bracci \& de Beeck, 2016) - defined by selecting the peak of activation on the IPS (Bracci \& de Beeck, 2016).

- Intraparietal Sulcus-Tool selective (IPS-Tool) - (Tools > Scrambled) (Bracci, et al., 2016; Bracci et al., 2016) - defined by selecting the peak of activation on the IPS (Bracci \& Op de Beeck, 2016).

- Supramarginal Gyrus (SMG) - (Tools > Scrambled) (Creem-Regehr, et al., 2007) defined by selecting the peak of activation located most anterior along the SMG (Peeters, et al., 2013), lateral to the anterior segment of the IPS (Gallivan, et al., 2013), posterior to the Precentral Suclus (PreCS) and superior to the lateral sulcus (Ariani, et al., 2015).

- Dorsal Premotor Cortex (PMd) - (Tools > Scrambled) - defined by selecting the peak of activation at the junction of the PreCS and the superior frontal sulcus (Gallivan et al., 2013; Ariani, et al., 2015). 
- Ventral Premotor Cortex (PMv) - (Tools > Scrambled) (Creem-Regehr, et al., 2007) defined by selecting the voxels inferior and posterior to the junction between the inferior frontal sulcus and the PreCS (Gallivan et al., 2013).

- Early Visual Cortex (EVC) - (All Conditions > Baseline) (Bracci \& de Beeck, 2016) defined by selecting the voxels in the occipital cortex near the calcarine sulcus (Singhal,

Pattern Classification. We performed MVPA independently for tool and non-tool trial types. Independent linear pattern classifiers (linear Support Vector Machine; SVM), were trained to learn the mapping between a set of brain-activity patterns (beta values computed from single blocks of activity) from the visual ROls and the type of grasp being performed with the tools (typical vs atypical) or non-tools (right vs left). To test the performance of our classifiers, decoding accuracy was assessed using an n-fold leave-one-run-out cross validation procedure; thus, our models were built from $n-1$ runs and were tested on the independent $n$th run (repeated for the $\mathrm{n}$ different possible partitions of runs in this scheme (Duda et al., 2001; Smith et al., 2010; 2015; Gallivan et al., 2016) before averaging across $n$ iterations to produce a representative decoding accuracy measure per participant and per ROI. Beta estimates for each voxel were normalised (separately for training and test data) within a range of -1 to +1 before input to the SVM (Chang \& Lin, 2011) and the linear SVM algorithm was implemented using the default parameters provided in the LibSVM toolbox $(C=1)$. Pattern classification was performed with a combination of in-house scripts (Smith et al., 2010; 2015) using Matlab with the Neuroelf toolbox (version 0.9c; http://neuroelf.net) and a linear SVM classifier (libSVM 2.12 toolbox;

381 https://csie.ntu.edu.tw/ cjlin/libsvm). 
383 Statistical Analysis. One-tailed one-sample t-tests were used to test for above chance decoding

384 for tool and non-tool action classifications in every ROI independently. If the pattern of results was consistent with our hypothesis (i.e., decoding accuracy was significantly above chance for tools, but not non-tools), we further ran a one-tailed pairwise t-tests to compare if decoding accuracy was significantly higher for tools than non-tools. Additionally, to test for differences in decoding accuracy between ROls we used repeated measures $2 \times 2$ ANOVAs with ROI (tool vs hand selective) and object category (tool vs non-tool) as within-subject factors. Then, to test if univariate differences would differ between grasp types for the tools, but not non-tools we ran $2 \mathrm{x}$ 2 ANOVAs with grasp type (typical/right vs atypical/left) and object category (tools vs non-tools) by entering mean beta weights for each ROI. Separately for each set of analyses we corrected

393 for multiple comparisons with False Discovery Rate (FDR) correction of $q \leq 0.05$ (Benjamini \& Hochberg, 1995; Benjamini \& Yekutieli, 2001) across the number of tests. Only significant results are reported (see Fig. 2). Our sample size was based on similar motor studies using MVPA (e.g., Ariani et al., 2015; 2018; Gallivan et al., 2009; 2013; 2014), though no power analysis was performed prior to data collection. supplemented null-hypothesis significance tests with Bayes factors (BF; Wagenmakers, 2007; Rouder et al., 2009). Bayes factors were estimated using the bayesFactor toolbox in Matlab (version 1.1; https://klabhub.github.io/bayesFactor). The Jeffreys-Zellner-Siow default prior on effect sizes was used (Rouder, Morey, Speckman, \& Province, 2012) and BF's were interpreted according to criteria set out by Jeffreys (1961; cited from Jarosz \& Wiley, 2014) where a BF 01 between 1-3 and > 3 indicates 'anecdotal' and 'substantial' evidence in favour of the null, respectively.

406

407 Data Availability. Stimuli, code for running experiment and for MVPA analyses and ROI data are 408 accessible from Open Science Framework at: https://osf.io/zxnpv. Full raw MRI dataset (real 409 action and visual localizer) is accessible from OpenNEURO at:

\section{0 https://openneuro.org/datasets/ds003342/versions/1.0.0.}




\section{Results}

413 In line with our predictions, as can be seen in Fig. 2, a one-sample t-test against chance (50\%)

414 showed that SVM decoding accuracy (FDR-corrected) from hand-selective ROIs in LOTC and

415 IPS were significantly greater-than-chance when discriminating typical vs atypical actions with 416 tools (LOTC-Hand accuracy $=56 \% \pm(\mathrm{SD}) 0.9 \%, t(16)=2.73, p=0.007, d=0.66$; IPS-Hand

417 accuracy $=57 \% \pm 0.11 \%, t(18)=2.72, p=0.007, d=0.62)$, but not biomechanically-matched

418 actions with non-tools (right vs left; LOTC-Hand: $p=0.252$, IPS-Hand: $p=0.844$ ). In fact, there 419 was substantial evidence in favour of null decoding of non-tool actions for the IPS ROI (LOTC-

420 Hand: $\mathrm{BF}_{01}=2.29$; IPS-Hand = 8.4). Importantly, results from a stringent between-classification 421 paired samples t-test also further supported this: typicality decoding accuracy from both LOTC-

422 Hand and IPS-Hand was significantly higher for tools than for biomechanically-matched actions 423 with non-tools (LOTC-Hand: $t(16)=2.11, p=0.026, d=0.51$; IPS-Hand: $t(18)=3.26, p=0.002$, $424 d=0.75$; Fig. 2A and Fig. 2B).

No other visual ROI, including tool-selective areas, displayed the same significant effects as hand-selective areas (Fig. 2A and Fig. 2B). For tool-selective ROls, decoding accuracy was not significantly greater-than-chance for classifying actions with tools or non-tools (all p's > 0.024), with the Bayesian approach demonstrating strong evidence in favour of the null for PMv (tool: $\mathrm{BF}_{01}=3.23$; non-tool: $\mathrm{BF}_{01}=6.85$ ) and $\mathrm{SMG}$ tool decoding (tool: $\mathrm{BF}_{01}=8.85$; other $\mathrm{BF}_{01}$ 's $<$ 1.08). The exception to this was tool-selective PMd which was found to decode significantly above chance actions with non-tools (accuracy $=59 \% \pm 0.08 \% t(13)=4.11, p=0.001, d=1.1$; Fig. $2 \mathrm{~A})$, but not tools $\left(\mathrm{BF}_{01}=4.42\right)$. As for object- and body-selective areas, LOTC-Object decoding accuracy did not differ from chance for tools or non-tools $(p>0.026)$, though evidence in favour of the null was anecdotal $\left(\mathrm{BF}_{01}\right.$ 's $\left.<1.33\right)$, whereas $\mathrm{pFs}$ and LOTC-Body decoded actions above chance with both tools (pFs: accuracy $=58 \% \pm 0.14 \% t(18)=2.57, p=0.01, d=$ 
0.59 ; LOTC-Body: accuracy $=59 \% \pm 0.08 \%(t(17)=4.75, p<0.001, d=1.12)$ and non-tools

(pFs: accuracy $=57 \% \pm 0.12 \% t(18)=2.59, p=0.009, d=0.59 ;$ LOTC-Body: accuracy $=56 \% \pm$

$4430.10 \%(t(17)=2.46, p=0.012, d=0.58$; Fig. $2 \mathrm{~A})$. Like many of the tool-selective ROls, the

444 control EVC ROI was not found to decode actions with either type of object ( $p$ 's $<0.026$ ), albeit evidence in favour of the null was anecdotal $\left(\mathrm{BF}_{01}\right.$ 's $\left.>0.37\right)$.

Since we obtained a different pattern of results for LOTC and IPS ROIs that were hand-

vs tool-selective, we compared the decoding accuracies between these regions with a repeated measures ANOVA with ROI (hands vs tool-selective) and object category (tool vs non-tools) as within-factors. As shown in Fig. 2B, there was a significant interaction between $\mathrm{ROI}$ and object category in IPS $\left(F(1,18)=5.94, p=0.025, \eta^{2}=0.25\right)$. Post-hoc t-tests showed that for IPS-Hand, grasp type decoding was significantly higher for tools than non-tools (mean difference $=0.1 \%$, $\mathrm{SE}=0.03 \% ; p=0.004$ ), but not for IPS-Tool (mean difference $=0.02 \%, \mathrm{SE}=0.03 \%$ ). However, for LOTC this interaction was not significant $(p=0.379$; Fig. $2 B)$, nor were the remaining main effects (all p's >0.367).

Next, we examined whether significant decoding in hand-selective cortex could be accounted for by low-level sensory differences between the tools' handles and functional-ends. First, to test the possibility that tool-specific decoding in hand-selective cortex could be driven by simple textural differences (e.g., a smooth handle vs a serrated knife blade), we repeated the analysis using a left somatosensory cortex ROI (SC; defined by selecting the peak voxel in the postcentral gyrus in the same subjects with an independent univariate contrast of All Grasps > Baseline; Fabbri et al., 2014, 2016). However, unlike the higher accuracies for grasping tools than non-tools in the hand-selective ROls, grasp type decoding in SC was significantly greaterthan-chance for both tool (accuracy $=57 \% \pm 0.11 \%, t(18)=3.04, p=0.004, d=0.7$ ) and nontools (accuracy $=57 \% \pm 0.09 \% t(18)=3.45, p=0.001, d=0.79$; Fig. $2 \mathrm{C}$ ). This indicates that tool-specific decoding in hand-selective cortex cannot be solely explained by somatosensory differences in the stimuli. Second, we tested if size differences between our objects, and thus grip size, could drive tool-specific decoding in hand-selective cortex (i.e., the functional-end of the tool being wider than its handle for the spoon and pizza cutter). As shown in Fig. 3A, we decoded smaller vs larger objects in three separate decoding analysis, regardless of whether the 
objects were tools or non-tools. Each separate grip size pair decoding analysis is shown in each row of images of Fig. 3A (from top to bottom: small vs medium; small vs large; medium vs large). Decoding accuracies for each grip size pair where then averaged and tested against chance using a one-tailed one-sample t-test. Decoding of grip size was not significant for any visual ROI (all p's $\geq 0.1$; Fig. 3B) and evidence in favour of the null was strong for most ROls including IPSHand $\left(\mathrm{BF}_{01}=8\right), \mathrm{EVC}\left(\mathrm{BF}_{01}=3.22\right)$, LOTC-Object $\left(\mathrm{BF}_{01}=4.93\right), \mathrm{pFs}\left(\mathrm{BF}_{01}=5.97\right), \mathrm{SMG}\left(\mathrm{BF}_{01}=\right.$ 3.33), $\mathrm{PMv}\left(\mathrm{BF}_{01}=3.91\right)$ and $\mathrm{PMd}\left(\mathrm{BF}_{01}=3.56\right.$; all other $\mathrm{BF}_{01}$ 's $\left.>1.84\right)$. Taken together, these findings suggest that hand-selective regions, particularly in the IPS, are sensitive to whether a tool is grasped correctly by its handle or not, and that these effects are not simply due to textural or size differences between the stimuli used or actions performed.

In addition, we found that the significant decoding accuracies reported here do not simply reflect the overall response amplitudes within each $\mathrm{ROI}$. When we analysed the mean beta weights in ANOVAs with grasp type and object category as within-subject factors for each ROI (i.e., as done in conventional univariate analysis; see Fig. 4), the only significant effect observed was a main effect of object category (unrelated to typicality), where greater activation was found for tools relative to non-tools in LOTC-Tool $\left(F(1,16)=9.25, p=0.008, \eta^{2}=0.37\right.$; mean difference $=0.1, \mathrm{SE}=0.03), \mathrm{pFs}\left(F(1,18)=8.68, p=0.009, \eta^{2}=0.33 ;\right.$ mean difference $\left.=0.07, \mathrm{SE}=0.02\right)$ and SMG $\left(F(1,16)=10.5, p=0.005, \eta^{2}=0.4\right.$; mean difference $=0.089$, SE $\left.=0.03\right)$.

\section{Discussion}

494 Our understanding of how the human brain represents object properties (Kanwisher, 2010) and simple hand movements (Gallivan \& Culham, 2015) has significantly advanced in the last few decades, however, far less is known about the neural representations that underpin real actions involving 3D tools (Valyear et al., 2017). Most neuroimaging experiments that investigate how tools and their associated actions are represented in the brain have used visual paradigms 
where objects and body-parts are displayed as 2D images (Ishibashi et al., 2016). These studies

500 have discovered a tight anatomical and functional relationship between hand- and tool-selective areas in LOTC and IPS, thought to reflect action-related processing, however this was yet to be directly tested (Bracci et al., 2012; 2013; 2016; Peelen et al., 2013; Striem-Amit et al., 2017; Maimon Mor, 2020). Here we defined visually category-selective areas and investigated if they were sensitive to real action affordances involving 3D tools. We found the first evidence that hand-selective cortex (left IPS-Hand and LOTC-Hand) represents whether a 3D tool is being grasped appropriately by its handle. Remarkably, the same effects were not observed in tool-, object-, or body-selective areas, even when these areas overlapped with hand-selective voxels in IPS and LOTC. process sensorimotor affordances of typicality for hand movements with 3D tools. Importantly,

511 these action-related representations were detected exclusively for actions with tools, but not for

512 biomechanically matched actions with non-tools. This tool-specificity was particularly evident in

513 IPS-hand because Bayesian evidence demonstrated that decoding of grasp type with non-tools

514 was not possible. In a similar vein, while the IPS ANOVA demonstrated boosted tool-specific 515 decoding specifically for the hand-selective ROI, this effect was not significant in LOTC. This suggests that typicality effects may be less robust for LOTC-hand. Our findings shed light into the

517 features of sensorimotor processing in hand-selective areas. First, their representations are

518 sensitive to concepts acquired through experience (i.e., knowing how to grasp tools appropriately

519 is a learnt skill; Martin, 2007), fitting with evidence showing that learning about how to manipulate

520 tools (Weisberg et al., 2007) or even performing such actions (Valyear et al., 2012; Brandi et al.,

521 2014; Styrkowiec et al., 2019) affects LOTC and IPS activity. For example, our results are compatible with those from Brandi et al. (2014) who showed coactivation of these regions during

523 'use' actions of tools/non-tools. Our results, however, additionally suggest that this learnt

524 information, at least for grasping, is coded in specific category-selective parts of LOTC and IPS.

525 Second, information processed by hand-selective cortex is represented in an abstract format

526 beyond low level properties (e.g., basic kinematics), since Bayesian evidence strongly suggested

527 that decoding grip size was not possible. This fits well with reports that hand-/tool-selective 
overlap exists in people born without vision (Peelen et al., 2013) or without hands (Striem-Amit et

529 al., 2017) suggesting that their development is driven by similarities in how they process non-

530 sensory tool information. In addition, our data also resonates with previous studies showing that

531 tool-selective areas in pMTG/LOTC and IPS represent abstract action goals (reach vs grasp)

532 regardless of biomechanics (Gallivan et al., 2013; Jacobs et al., 2010), albeit our findings were

533 observed for hand-selective areas only. Third, our study shows that these high-level

534 representations are automatically evoked (Valyear et al., 2012) as throughout the real-action

$535 \mathrm{fMRI}$ task there was no explicit requirement to use the tools and participants were never told that we were investigating 'tools'. Here we demonstrate that these principles, frequently described to support tool-use (Gibson, 1979; Imamizu et al., 2003; Maravita \& Iriki 2004; Umilta et al., 2008; Lingnau \& Downing, 2015), apply to brain areas specialised for representing the human hand, our primary tool for interacting with the world.

An intriguing aspect of our results is that typicality decoding was successful using activity patterns from hand-selective, but not overlapping parts of tool-selective cortex in the LOTC and IPS. Bayesian evidence only anecdotally supported the possibility that decoding was null from tool-selective areas, but significantly stronger typicality decoding was observed for IPS-Hand

544 than IPS-Tool during tool, but not non-tool grasps. In contrast to previous picture viewing fMRI studies showing that overlapping hand- and tool-selective regions exhibit similar responses

546 (Bracci et al., 2012; 2013; 2016), our findings uniquely support previous speculations that hand-

547 selective IPS, and possibly LOTC, could be functionally distinct from tool-selective regions 548 despite their anatomical overlap (e.g., Striem-Amit et al., 2017). This pattern of results is unlikely

549 to be driven by differences in $\mathrm{ROI}$ radius (Etzel et al., 2013) since voxel size differences were 550 negligible between hand- and tool-selective ROIs (mean difference: IPS: 29; LOTC: 4). In fact, if category-related results were merely caused by ROI size, then significant decoding should have also been observed in the much larger LOTC-Object ROI (see Table 1). Alternatively, successful higher decoding in hand than tool-selective areas might reflect that our task simply required grasping-to-touch the tools, rather than their utilisation. That is, coding in category-selective areas might operate in an effector-dependent manner, akin to how tool-selective pMTG/LOTC 
557 by the hand alone (Gallivan et al., 2013). In line with this interpretation, neural representations in

558 LOTC-Hand of one-handed amputees are also known to become richer as prosthetic usage 559 increases (Van den Heiligenberg 2018), which, again, indicates that the representations in hand-

560 selective cortex depend on effector use. An alternative, but not mutually exclusive, possibility is

561 that only tool-use actions elicit tool-selective representations (see Randerath et al., 2010)

562 because of the cognitively taxing demands these complex actions rely on, such as retrieving

563 knowledge about manipulation hierarchies (Buxbaum, 2017) or the laws that constrain object

564 movement (Fischer et al., 2016). In either case, the specificity of decoding typical tool grasps in

565 hand-, rather than tool- and hand-, selective cortex challenges the popular interpretation that

566 brain activation for viewing tool images is a reflection of sensorimotor processing linked to tool

567 manipulation (Martin et al., 1996; Mahon et al., 2007; Fang \& He, 2005; Grafton et al., 1997;

568 Martin \& Chao, 2001; also see Mahon \& Caramazza, 2009).

$569 \quad$ There are several differences between our study and previous research. First our

570 univariate analysis revealed no relationship between mean activity and typicality. Previous

571 studies have found greater univariate activation in occipito-temporal and/or fronto-parietal cortex

572 for typical relative to atypical actions when participants viewed pictures and movies or

573 pantomimed (Johnson-Frey et al., 2003; Valyear \& Culham, 2010; Yoon et al., 2012; Mizelle et

574 al., 2013; Przybylski \& Króliczak, 2017). Our results fit the claim that MVPA can reveal fine-

575 grained effects (Kriegeskorte, et al., 2006), as recently argued by Buchwald et al. (2018) when

576 showing that pantomimed typical tool vs non-tool grasps could be decoded from a range of

577 regions including premotor and intraparietal areas. We suspect that task differences are also an

578 important contributing factor to the general lack of univariate effects. For example, our

579 experiment involved fewer, less varied, exemplars than in these previous picture studies.

580 Likewise, our grasp-to-touch paradigm is simpler than studies showing greater univariate

581 activations in the left SMG, premotor cortex, LOTC and IPS when performing real tool-use

582 actions (Brandi et al., 2014; Valyear et al., 2012) or haptically-guided typical tool grasps

583 (Styrkowiec et al., 2019) relative to tool/non-tool control actions. Finally, in our study, grasping

584 always involved a precision grip whereas previous studies employed power grasps which are

585 better suited for certain actions with some specific tools. This factor may have led to the lack of 
typicality decoding effects in tool-selective cortex as these areas could be sensitive to both the

587 side of the object being grasped and the function of particular grips (Buxbaum et al., 2006). We designed our precision grasping task to investigate tool affordances while carefully equating biomechanics between actions, such that decoding typicality was unlikely to be attributed to motor-related differences. Future real action studies manipulating the type of grasp (e.g., grasp vs use) are needed to further identify the content of information coded by visual hand-/toolselective areas. It is worth noting that we were unable to match the visual symmetry between object categories (our tools were asymmetric while the non-tools were symmetric) because asymmetric non-tool bars were perceived as tools by participants (i.e., the wider side perceived as a functional-end). Nonetheless, tool-specific decoding in hand-selective cortex is unlikely to be explained by simple effects of symmetry: if effects were related to symmetry comparable decoding effects should have been observed in symmetry-responsive regions (e.g., LOTC-

599 Object; EVC; Beck, et al., 2006), particularly since they are also known to code motor-related information (e.g., Gallivan \& Culham, 2015; Monaco et al., 2020). In conclusion, parietal and occipital visual regions specialised for representing hands were found to encode information about the functional relationship between the grasping hand and a tool, implicating hand-selective cortex in motor control. These findings raise novel questions about the possibility that overlapping hand- and tool-selective regions are functionally distinct and begin to uncover which brain regions evolved to support tool-use, a defining feature of our species.

607

\section{REFERENCES}

609 Ambrose, S. H. (2001). Paleolithic technology and human evolution. Science, 291(5509), 17481753.

611 Ariani, G., Oosterhof, N. N., \& Lingnau, A. (2018). Time-resolved decoding of planned delayed 612 and immediate prehension movements. Cortex, 99, 330-345.

613 Ariani, G., Wurm, M. F., \& Lingnau, A. (2015). Decoding internally and externally driven movement plans. J Neurosci, 35(42), 14160-14171. 
615 Astafiev, S. V., Stanley, C. M., Shulman, G. L., \& Corbetta, M. (2004). Extrastriate body area in

616 human occipital cortex responds to the performance of motor actions. Nat Neurosci, 7(5), $617 \quad 542-548$

618 Bach, P., Peelen, M. V., \& Tipper, S. P. (2010). On the role of object information in action 619 observation: an fMRI study. Cereb Cortex, 20(12), 2798-2809.

620 Beck, D. M., Pinsk, M. A., \& Kastner, S. (2005). Symmetry perception in humans and macaques. $621 \quad$ Trends Cogn Sci, 9(9), 405-406.

622 Benjamini, Y., \& Hochberg, Y. (1995). Controlling the false discovery rate: a practical and 623 powerful approach to multiple testing. Journal of the Royal statistical society: Series B (Methodological), 57(1), 289-300.

Benjamini, Y., \& Yekutieli, D. (2001). The control of the false discovery rate in multiple testing under dependency. Ann Stat, 1165-1188.

Birn, R. M., Cox, R. W., \& Bandettini, P. A. (2004). Experimental designs and processing strategies for fMRI studies involving overt verbal responses. Neuroimage, 23(3), 10461058.

Boynton, G. M., Engel, S. A., Glover, G. H., \& Heeger, D. J. (1996). Linear systems analysis of functional magnetic resonance imaging in human V1. J Neurosci, 16(13), 4207-4221.

Bracci, S., \& de Beeck, H. O. (2016). Dissociations and associations between shape and category representations in the two visual pathways. J Neurosci, 36(2), 432-444.

634 Bracci, S., \& Peelen, M. V. (2013). Body and object effectors: the organization of object representations in high-level visual cortex reflects body-object interactions. J Neurosci, 33(46), 18247-18258.

637 Bracci, S., Caramazza, A., \& Peelen, M. V. (2018). View-invariant representation of hand postures in the human lateral occipitotemporal cortex. Neurolmage, 181, 446-452. overlapping responses to tools and hands in left lateral occipitotemporal cortex. $\mathrm{J}$ Neurophysiol, 107(5), 1443-1456. 
Bracci, S., letswaart, M., Peelen, M. V., \& Cavina-Pratesi, C. (2010). Dissociable neural responses to hands and non-hand body parts in human left extrastriate visual cortex. $\mathrm{J}$ Neurophysiol, 103(6), 3389-3397.

645 Brainard, D.H. (1997). The Psychophysics Toolbox. Spa Vis, 10(4), 433-436.

646 Brandi, M. L., Wohlschläger, A., Sorg, C., \& Hermsdörfer, J. (2014). The neural correlates of planning and executing actual tool use. J Neurosci, 34(39), 13183-13194.

648 Buxbaum, L. J. (2017). Learning, remembering, and predicting how to use tools: Distributed neurocognitive mechanisms: Comment on Osiurak and Badets (2016). Psychol Rev, 124(3), 346-360.

Buxbaum, L. J., Kyle, K. M., Tang, K., \& Detre, J. A. (2006). Neural substrates of knowledge of hand postures for object grasping and functional object use: Evidence from fMRI. Brain research, 1117(1), 175-185.

Chang, C. C., \& Lin, C. J. (2011). LIBSVM: A library for support vector machines. ACM transactions on intelligent systems and technology (TIST), 2(3), 1-27.

Creem-Regehr, S. H., Dilda, V., Vicchrilli, A. E., Federer, F., \& Lee, J. N. (2007). The influence of complex action knowledge on representations of novel graspable objects: evidence from functional magnetic resonance imaging. J Int Neuropsych Soc, 13(6), 1009-1020.

Culham, J. C. (2006). Functional neuroimaging: Experimental design and analysis R. Cabeza, A.

662 Damasio, H. (1995). Human brain anatomy in computerized images. New York: Oxford University Press.

664 Dinstein, I., Thomas, C., Behrmann, M., \& Heeger, D. J. (2008). A mirror up to nature. Curr Biol, 665 18(1), R13-R18.

Duda, R. O., Hart, P. E., \& Stork, D. G. (2001). Pattern classification. Int J Computational Intelligence and Applications, 1, 335-339.

668 Etzel, J. A., Zacks, J. M., \& Braver, T. S. (2013). Searchlight analysis: promise, pitfalls, and potential. Neuroimage, 78, 261-269. 
670 Fabbri, S., Strnad, L., Caramazza, A., \& Lingnau, A. (2014). Overlapping representations for grip type and reach direction. Neuroimage, 94, 138-146.

672 Fabbri, S., Stubbs, K. M., Cusack, R., \& Culham, J. C. (2016). Disentangling representations of object and grasp properties in the human brain. J Neurosci, 36(29), 7648-7662.

674 Fang, F., \& He, S. (2005). Cortical responses to invisible objects in the human dorsal and ventral pathways. Nat Neurosci, 8(10), 1380-1385.

Fernández-Espejo, D., Rossit, S., \& Owen, A. M. (2015). A thalamocortical mechanism for the absence of overt motor behavior in covertly aware patients. JAMA neurology, 72(12), 1442-1450.

Fischer, J., Mikhael, J. G., Tenenbaum, J. B., \& Kanwisher, N. (2016). Functional neuroanatomy of intuitive physical inference. Proc Natl Acad Sci USA, 113(34), E5072-E5081.

Frey, S. H. (2007). What puts the how in where? Tool use and the divided visual streams hypothesis. Cortex, 43(3), 368-375.

Frey, S. H. (2008). Tool use, communicative gesture and cerebral asymmetries in the modern human brain. Philos Trans R Soc Lond B Biol Sci, 363(1499), 1951-1957.

Gallivan, J. P., Cant, J. S., Goodale, M. A., \& Flanagan, J. R. (2014). Representation of object weight in human ventral visual cortex. Curr Biol, 24(16), 1866-1873.

687 Gallivan, J. P., Cavina-Pratesi, C., \& Culham, J. C. (2009). Is that within reach? fMRI reveals that the human superior parieto-occipital cortex encodes objects reachable by the hand. $\mathrm{J}$ Neurosci, 29(14), 4381-4391.

Gallivan, J. P., \& Culham, J. C. (2015). Neural coding within human brain areas involved in

692 Gallivan, J. P., Johnsrude, I. S., \& Randall Flanagan, J. (2016). Planning ahead: object-directed sequential actions decoded from human frontoparietal and occipitotemporal networks. Cerebral Cortex, 26(2), 708-730. mechanisms of human tool use. Elife, 2, e00425.

697 Gibson J. J. (1979) The ecological approach to visual perception. Boston: Houghton Mifflin. 
Grafton, S. T., Fadiga, L., Arbib, M. A., \& Rizzolatti, G. (1997). Premotor cortex activation during observation and naming of familiar tools. Neuroimage, 6(4), 231-236.

Grèzes, J., Tucker, M., Armony, J., Ellis, R., \& Passingham, R. E. (2003). Objects automatically potentiate action: an fMRI study of implicit processing. Eur J Neurosci, 17(12), 2735-

Grill-Spector, K., Kourtzi, Z., \& Kanwisher, N. (2001). The lateral occipital complex and its role in

Grill-Spector, K., Kushnir, T., Edelman, S., Avidan, G., Itzchak, Y., \& Malach, R. (1999).

706 Differential processing of objects under various viewing conditions in the human lateral occipital complex. Neuron, 24(1), 187-203.

708

Hutchison, R. M., Culham, J. C., Everling, S., Flanagan, J. R., \& Gallivan, J. P. (2014). Distinct 709 and distributed functional connectivity patterns across cortex reflect the domain-specific constraints of object, face, scene, body, and tool category-selective modules in the ventral visual pathway. Neuroimage, 96, 216-236.

Imamizu, H., Kuroda, T., Miyauchi, S., Yoshioka, T., \& Kawato, M. (2003). Modular organization of internal models of tools in the human cerebellum. Proc Natl Acad Sci USA, 100(9),

Ishibashi, R., Pobric, G., Saito, S., \& Lambon Ralph, M. A. (2016). The neural network for toolrelated cognition: an activation likelihood estimation meta-analysis of 70 neuroimaging contrasts. Cogn Neuropsychol, 33(3-4), 241-256.

Jacobs, S., Danielmeier, C., \& Frey, S. H. (2010). Human anterior intraparietal and ventral premotor cortices support representations of grasping with the hand or a novel tool. $\mathrm{J}$

Jarosz, A. F., \& Wiley, J. (2014). What are the odds? A practical guide to computing and Cogn Neurosci, 22(11), 2594-2608. reporting Bayes factors. J Probl Solving, 7(1), 2.

Johnson-Frey, S. H., et al., (2003). Actions or hand-object interactions? Human inferior frontal cortex and action observation. Neuron, 39(6), 1053-1058.

Kanwisher, N. (2010). Functional specificity in the human brain: a window into the functional architecture of the mind. Proc Natl Acad Sci USA, 107(25), 11163-11170. 
Kay, K. N., Naselaris, T., Prenger, R. J., \& Gallant, J. L. (2008). Identifying natural images from human brain activity. Nature, 452(7185), 352-355.

Konkle, T., \& Caramazza, A. (2013). Tripartite organization of the ventral stream by animacy and object size. J Neurosci, 33(25), 10235-10242.

Kriegeskorte, N., Goebel, R., \& Bandettini, P. (2006). Information-based functional brain mapping. Proc Natl Acad Sci USA, 103(10), 3863-3868.

Lewis, J. W. (2006). Cortical networks related to human use of tools. The neuroscientist, 12(3), 211-231.

Lingnau, A., \& Downing, P. E. (2015). The lateral occipitotemporal cortex in action. Trends Cogn Sci, 19(5), 268-277.

Mahon, B. Z., \& Caramazza, A. (2009). Concepts and categories: a cognitive neuropsychological perspective. Annu Rev Psychol, 60, 27-51.

Mahon, B. Z., Milleville, S. C., Negri, G. A., Rumiati, R. I., Caramazza, A., \& Martin, A. (2007). Action-related properties shape object representations in the ventral stream. Neuron, 55(3), 507-520.

Maimon Mor, R. O., \& Makin, T. R. (2020). Is an artificial limb embodied as a hand? Brain decoding in prosthetic limb users. PLoS Biol, 18(6), e3000729.

Malach, R., Reppas, J. B., Benson, R. R., Kwong, K. K., Jiang, H., et al. (1995). Object-related activity revealed by functional magnetic resonance imaging in human occipital cortex. Proc Natl Acad Sci USA, 92(18), 8135-8139.

Maravita, A., \& Iriki, A. (2004). Tools for the body (schema). Trends Cogn Sci, 8(2), 79-86.

Martin, A. (2007). The representation of object concepts in the brain. Annu. Rev. Psychol., 58, 25-45.

Martin, A. (2016). GRAPES—Grounding representations in action, perception, and emotion systems: How object properties and categories are represented in the human brain. Psychon Bull Rev, 23(4), 979-990.

Martin, A., \& Chao, L. L. (2001). Semantic memory and the brain: structure and processes. Curr Opin Neurobiol, 11(2), 194-201. 
Martin, A., Wiggs, C. L., Ungerleider, L. G., \& Haxby, J. V. (1996). Neural correlates of categoryspecific knowledge. Nature, 379(6566), 649-652.

Mizelle, J. C., Kelly, R. L., \& Wheaton, L. A. (2013). Ventral encoding of functional affordances: a neural pathway for identifying errors in action. Brain and cognition, 82(3), 274-282.

Monaco, S., Malfatti, G., Culham, J. C., Cattaneo, L., \& Turella, L. (2020). Decoding motor imagery and action planning in the early visual cortex: overlapping but distinct neural mechanisms. Neurolmage, 116981.

Monaco, S., Sedda, A., Cavina-Pratesi, C., \& Culham, J. C. (2015). Neural correlates of object size and object location during grasping actions. European J Neurosci, 41(4), 454-465.

Mur, M., Bandettini, P. A., \& Kriegeskorte, N. (2009). Revealing representational content with pattern-information fMRI—an introductory guide. Soc Cogn Affect Neurosci, 4(1), 101109.

Oldfield, R.C. (1971). The assessment and analysis of handedness: The Edinburgh inventory. Neuropsychologia, 9(1), 97-113.

Palser, E., \& Cavina-Pratesi, C. (2018). Left lateral occipito-temporal cortex encodes compatibility between hands and tools: an fMRI adaptation study. 10.31234/osf.io/kbjw4 (15 June 2019).

Peelen, M. V., \& Downing, P. E. (2005). Is the extrastriate body area involved in motor actions? Nat Neurosci, 8(2), 125-125.

Peelen, M. V., et al., (2013). Tool selectivity in left occipitotemporal cortex develops without vision. J Cogn Neurosci, 25(8), 1225-1234.

Peeters, R. R., Rizzolatti, G., \& Orban, G. A. (2013). Functional properties of the left parietal tool use region. Neuroimage, 78, 83-93.

Perini, F., Caramazza, A., \& Peelen, M. V. (2014). Left occipitotemporal cortex contributes to the discrimination of tool-associated hand actions: fMRI and TMS evidence. Front Hum

Przybylski, Ł., \& Króliczak, G. (2017). Planning functional grasps of simple tools invokes the handindependent praxis representation network: an fMRI study. J Int Neuropsychol Soc, 23(2), 108-120. 
784 Randerath, J., Goldenberg, G., Spijkers, W., Li, Y., \& Hermsdörfer, J. (2010). Different left brain regions are essential for grasping a tool compared with its subsequent use. Neuroimage, 53(1), 171-180.

Randerath, J., Goldenberg, G., Spijkers, W., Li, Y., \& Hermsdörfer, J. (2011). From pantomime to actual use: how affordances can facilitate actual tool-use. Neuropsychologia, 49(9), $2410-2416$.

Rossit, S., McAdam, T., Mclean, D. A., Goodale, M. A., \& Culham, J. C. (2013). fMRI reveals a lower visual field preference for hand actions in human superior parieto-occipital cortex (SPOC) and precuneus. Cortex, 49(9), 2525-2541.

Rouder, J. N., Morey, R. D., Speckman, P. L., \& Province, J. M. (2012). Default Bayes factors for ANOVA designs. J Math Psychol, 56(5), 356-374.

Rouder, J. N., Speckman, P. L., Sun, D., Morey, R. D., \& Iverson, G. (2009). Bayesian t tests for accepting and rejecting the null hypothesis. Psychon Bull Rev, 16(2), 225-237.

Sakuraba, S., Sakai, S., Yamanaka, M., Yokosawa, K., \& Hirayama, K. (2012). Does the human dorsal stream really process a category for tools? J. Neurosci. 32, 3949-3953.

Santello, M., Flanders, M., \& Soechting, J. F. (1998). Postural hand synergies for tool use. J Neurosci, 18(23), 10105-10115.

Singhal, A., Monaco, S., Kaufman, L. D., \& Culham, J. C. (2013). Human fMRI reveals that delayed action re-recruits visual perception. PLoS One, 8(9), e73629

Smith, F. W., \& Muckli, L. (2010). Nonstimulated early visual areas carry information about surrounding context. Proc Natl Acad Sci USA, 107(46), 20099-20103.

Smith, F.W. \& Goodale, M.A. (2015). Decoding visual object categories in early somatosensory cortex. Cereb Cortex, 25(4), 1020-1031.

807 Snow, J. C., et al., (2011). Bringing the real world into the fMRI scanner: Repetition effects for 808 pictures vs real objects. Sci Rep, 1, 130.

809 Striem-Amit, E., Vannuscorps, G., \& Caramazza, A. (2017). Sensorimotor-independent 810 development of hands and tools selectivity in the visual cortex. Proc Natl Acad Sci USA, $811 \quad$ 114(18), 4787-4792. 
812 Styrkowiec, P. P., Nowik, A. M., \& Króliczak, G. (2019). The neural underpinnings of haptically

813 guided functional grasping of tools: an fMRI study. Neuroimage, 194, 149-162.

814 Talairach, J. \& Tournoux, P. (1988). Co-Planar Stereotaxic Atlas of the Human Brain. Thieme,

$815 \quad$ New York.

816 Umiltà, M. A., Intskirveli, I., Grammont, F., Rochat, M., Caruana, F., Jezzini, A., Gallese, V., \&

817 Rizzolatti, G. (2008). When pliers become fingers in the monkey motor system. Proc Natl

$818 \quad$ Acad Sci USA, 105(6), 2209-2213.

819 Valyear, K. F., Cavina-Pratesi, C., Stiglick, A. J., \& Culham, J. C. (2007). Does tool-related fMRI

820 activity within the intraparietal sulcus reflect the plan to grasp? Neuroimage, 36, 94-108.

821 Valyear, K. F., \& Culham, J. C. (2010). Observing learned object-specific functional grasps

822 preferentially activates the ventral stream. J Cogn Neurosci, 22(5), 970-984.

823 Valyear, K. F., Fitzpatrick, A. M., \& McManus, E. F. (2017). "The neuroscience of human tool

824 use" in Evolution of Nervous Systems. Kaas, J. (Ed.), pp. 341-353, Elsevier, Oxford.

825 Valyear, K. F., Gallivan, J. P., McLean, D. A., \& Culham, J. C. (2012). fMRI repetition

826 suppression for familiar but not arbitrary actions with tools. J Neurosci, 32(12), 4247-

$827 \quad 4259$.

828 Van den Heiligenberg, F. M., et al., (2018). Artificial limb representation in amputees. Brain, $829 \quad 141(5), 1422-1433$.

830 Wagenmakers, E. J. (2007). A practical solution to the pervasive problems of $p$ values. Psychon $831 \quad$ Bull Rev, 14(5), 779-804.

832 Weisberg, J., Van Turennout, M., \& Martin, A. (2007). A neural system for learning about object 833 function. Cereb Cortex, 17(3), 513-521.

834 Yoon, E. Y., Humphreys, G. W., Kumar, S., \& Rotshtein, P. (2012). The neural selection and 835 integration of actions and objects: an fMRI study. J Cogn Neurosci, 24(11), 2268-2279. 


\section{Figure Legends}

837 Figure 1. Experimental set-up and design. (A) 3D-printed tool and non-tool control object pairs 838 (black markers on objects indicate grasp points) which were matched for elongation, width and

839 depth such that tool and non-tool actions were biomechanically similar. (B) Side view of real 840 action participant set-up used to present 3D objects at grasping distance (without the use of 841 mirrors). Red star indicates fixation LED. The hand is shown at its starting position. (C) Timing 842 and grasping tasks from subject's point of view for the real action experiment. During the 10s

843 ON-block the object was illuminated 5 times cueing the participant to grasp the object each time

844 by its left or right side (as per preceding auditory cue) with the right hand. Exemplar videos of trial

845 types can be accessed here: https://osf.io/gsmyw/. This was followed by a 10s OFF-block 846 involving no stimulation where the workspace remained dark. For MVPA, we treated tool and 847 non-tool trials independently, where for the tools only, right- and left-sided grasps were typical

848 and atypical grasps respectively (based on handle orientation). (D) Timing of visual localizer 849 experiment. In the visual localizer, blocks of tools, hands, chairs, bodies and scrambled 2D 850 image stimuli were presented in between fixation-only screens. (E) For each individual participant 851 independent ROls were defined for MVPA using functional activity from the visual localizer

852 (Table 1). The representative ROI locations are displayed on a group activation contrast map 853 from the visual localizer (All conditions $>\left(\right.$ Baseline $\left.{ }^{\star} 5\right)$ ) projected onto a left hemisphere cortical

854 surface reconstruction of a reference brain (COLIN27 Talairach) available from the neuroElf 855 package (http://neuroelf.net).

856

857 Figure 2. Grasp type decoding results in left hemisphere ROls. (A) Violin plots of MVPA data 858 from visual localizer ROIs for the typical vs atypical classification of grasping tools (white violins) 859 and, non-tool control grasping (right vs left decoding; grey violins). Box plot centre lines are mean

860 decoding accuracy while their edges and whiskers show \pm 1 SD and \pm 2 SEM, respectively.

861 Decoding accuracies of typical vs atypical grasping in IPS and LOTC hand-selective cortex (pink) 862 are significantly greater-than-chance for tools, but not non-tools. (B) ANOVA results comparing 863 the difference of decoding accuracy between tools (typical vs atypical) and non-tools (right vs 864 left) for the partially overlapping hand- and tool-selective ROls within the IPS and LOTC. (C) 
865 Violin plot of MVPA data for control ROI in somatosensory cortex (SC) based on an independent

866 contrast (all actions > baseline) from real action experiment showing significant decoding of

867 grasp type for both tools and non-tools. Red asterisks show FDR-corrected results while black

868 asterisks show uncorrected results.

869

870 Figure 3. Grip size decoding. (A) We decoded smaller vs larger objects in three separate

871 decoding analysis, regardless of whether the objects were tools or non-tools. Each separate grip

872 size pair decoding analysis is shown in each row of images of Fig.3A (from top to bottom: small

873 vs. medium; small vs. large; medium vs. large). The heads of the knife, spoon and pizzacutter

874 tools and their paired non-tools had matched small, medium and large widths, respectively.

875 Decoding accuracies for each grip size pair were then averaged and tested against chance using

876 a one-tailed one-sample t-test. In all cases, object category was collapsed to maximise power

877 and generalisability (i.e., grasping tools and non-tools), and reach direction was matched to

878 minimise kinematic variance (i.e., all actions were leftward). (B) Mean decoding accuracy in

879 visual localizer ROls for the small versus large classification collapsed across object category.

880 Error bars represent \pm 1 SEM.

881

882 Figure 4. Mean activation ( $\beta$ ) per ROI and condition used as input for pattern classification and

883 univariate analyses. Error bars represent \pm 1 SEM.

884

885

886

887 
888 Table 1. Visual Localizer ROI descriptives. ROI subject counts with their mean sizes (voxels) and 889 peak coordinates (Talairach).

\begin{tabular}{lccccc} 
& N Subjects & Mean size & \multicolumn{2}{c}{ Mean peak coordinates } \\
& with ROI & $(\mathrm{SD})$ & \multicolumn{2}{c}{ (SD) } \\
\cline { 3 - 5 } & & & $-14(6)$ & $-89(4)$ & $-9(9)$ \\
\hline EVC & 19 & $114(35)$ & $-42(4)$ & $-77(4)$ & $-7(4)$ \\
LOTC-Object & 19 & $148(34)$ & $-45(3)$ & $-76(5)$ & $2(6)$ \\
LOTC-Body & 18 & $55(30)$ & $-47(4)$ & $-71(4)$ & $-1(5)$ \\
LOTC-Hand & 17 & $81(44)$ & $-47(5)$ & $-71(5)$ & $-2(6)$ \\
LOTC-Tool & 17 & $77(45)$ & $-45(4)$ & $-57(3)$ & $3(4)$ \\
pMTG & 17 & $96(48)$ & $-40(4)$ & $-54(4)$ & $-14(4)$ \\
pFs & 19 & $105(41)$ & $-53(6)$ & $-28(4)$ & $27(6)$ \\
SMG & 17 & $69(43)$ & $-38(4)$ & $-46(7)$ & $42(3)$ \\
IPS-Hand & 19 & $110(57)$ & $-37(5)$ & $-41(7)$ & $42(5)$ \\
IPS-Tool & 19 & $81(55)$ & $-45(7)$ & $-1(6)$ & $31(5)$ \\
PMv & 14 & $61(42)$ & $-29(5)$ & $-13(4)$ & $51(4)$ \\
PMd & 14 & $47(28)$ & & & \\
\hline
\end{tabular}

